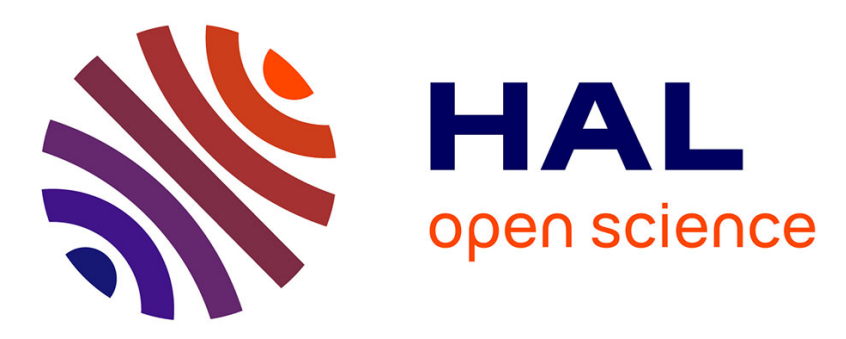

\title{
Effects of Voluntary Heart Rate Control on User Engagement in Virtual Reality
}

Samory Houzangbe, Olivier Christmann, Geoffrey Gorisse, Simon Richir

\section{To cite this version:}

Samory Houzangbe, Olivier Christmann, Geoffrey Gorisse, Simon Richir. Effects of Voluntary Heart Rate Control on User Engagement in Virtual Reality. 2019 IEEE Conference on Virtual Reality and 3D User Interfaces (VR), Mar 2019, Osaka, Japan. pp.2, 10.1109/VR.2019.8797759 . hal-02303892

\section{HAL Id: hal-02303892 \\ https://hal.science/hal-02303892}

Submitted on 2 Oct 2019

HAL is a multi-disciplinary open access archive for the deposit and dissemination of scientific research documents, whether they are published or not. The documents may come from teaching and research institutions in France or abroad, or from public or private research centers.
L'archive ouverte pluridisciplinaire HAL, est destinée au dépôt et à la diffusion de documents scientifiques de niveau recherche, publiés ou non, émanant des établissements d'enseignement et de recherche français ou étrangers, des laboratoires publics ou privés. 


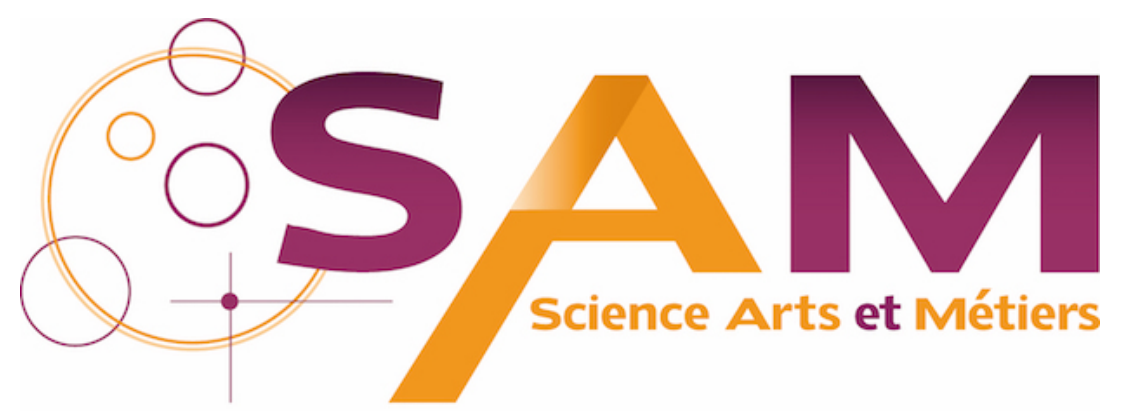

Archive Ouverte - Open Repository

\section{Science Arts \& Métiers (SAM)}

is an open access repository that collects the work of Arts et Métiers ParisTech researchers and makes it freely available over the web where possible.

This is an author-deposited version published in: https://sam.ensam.eu Handle ID: .http://hdl.handle.net/null

\section{To cite this version :}

Samory HOUZANGBE, Olivier CHRISTMANN, Geoffrey GORISSE, Simon RICHIR - Effects of Voluntary Heart Rate Control on User Engagement in Virtual Reality - In: 2019 IEEE Conference on Virtual Reality and 3D User Interfaces (VR), Japon, 2019-03-23 - Effects of Voluntary Heart Rate Control on User Engagement in Virtual Reality - 2019 


\section{Effects of Voluntary Heart Rate Control on User Engagement in Virtual Reality}

\author{
Samory Houzangbe* \\ Arts et Métiers ParisTech \\ EON Reality SAS
}

\author{
Olivier Christmann ${ }^{\dagger}$ \\ Arts et Métiers ParisTech
}

\author{
Geoffrey Gorisse \\ Arts et Métiers ParisTech
}

\author{
Simon Richir§ \\ Arts et Métiers ParisTech
}

\begin{abstract}
The usage of biofeedback in Virtual Reality (VR) is becoming more and more important in providing fully immersive experiences. With the rapid evolution of physiological monitoring technologies it is important to study how different modalities of biofeedback can alter user experience. While previous studies use biofeedback as an additional interaction mechanic. We created a protocol to assess heart rate control competency and used the results of said protocol to immerse our participants in a VR experience where the biofeedback mechanics are mandatory to complete a game. We observed consistent results between our competency scale and the participants' mastery of the biofeedback game mechanic in the VR experience. We also found that the biofeedback mechanic has a significant impact on engagement.
\end{abstract}

Keywords: Virtual Reality, Biofeedback, Engagement, User Study Index Terms: Human-centered computing-Interaction paradigms - Virtual reality; Human-centered computing-Interaction design process and methods-User centered design

\section{INTRODUCTION}

The use of physiology in Virtual Reality (VR) has been studied in order to bring more out of the experience. The general consensus seems to point toward the fact that the usage of physiological biofeedback can bring more user engagement in VR experiences [5]. However, biofeedback generally takes the role of an additional mechanic that the user can neglect [2]. That is why we want to study how a voluntary biofeedback game mechanic can influence user experience in Immersive Virtual Environments (IVE). Thus, it is important to provide a personalized experience, based on competency, and study how mandatory biofeedback affects user experience.

The mechanics of voluntary heart rate control have been thoroughly studied, notably by Sroufe [6]. The results of his studies show that it is possible to influence one's own heart rate with breathing and that a training strategy facilitate the acquisition of mastery over physiological control. Moreover Larkin et al. [3] demonstrate that the inclusion of biofeedback, coupled with training, increase even more the learning of heart rate control.

In order to study the effect of a voluntary heart rate biofeedback on user engagement in VR, we conduct a two-part study. The first part consists in determining the ability of the participants to control their own heart rate and classifying them in different skill groups. The second part of our experience exposes the participants to an IVE where they have to control their heart rate to complete the game. We analyze the participants reported feeling of engagement in regard to their competency.

\footnotetext{
*E-mail: samory.houzangbe@ensam.eu

†E-mail: olivier.christmann@ensam.eu

†E-mail: geoffrey.gorisse@ensam.eu

${ }^{\S}$ E-mail: simon.richir@ensam.eu
}

\section{Voluntary heART RATE CONTROL VR Game}

To quantify one's own heart rate control competency we developed a simple desktop application that proposes a series of heart rate control exercises consisting in accelerating or lowering the heart rate during the longest possible period of time, each exercise last 1:30 minutes. The application is split in three parts. The first is a blank screen informing the participants that the calibration of their heart rate baseline is being performed (based on the work of Houzangbe et al. [2]). The second part is a training phase, the screen shows different breathing patterns that the participants have to follow (a fast and shallow breathing pattern and a slow a deep breathing pattern), in order to observe the effects of these patterns on their heart rate. Finally, the experiment begins. It consists in a series of 8 exercises where the participants have to either increase their heart rate or lower it (inspired by the work of Manuck et al. [4]). During an exercise the participants have to maintain, for the maximum of time, their heart rate in a designated zone, based on their baseline value. Fifty-seven participants completed this experiment.

To measure how well the participants fare in the different conditions we calculated the mean time they spent in the designated heart rate zone for each round of the trials. Based on those time values we calculate time intervals that allow us to classify the participants in categories ranging from 1 (weak) to 4 (strong) for each trial type and compute a mean value of those scores to have a definite result on the 57 participants' ability: (1) 21 didn't succeed in controlling their heart rate, (2) 2 are considered weak at controlling their heart rate, (3) 13 are considered regular at controlling their heart rate, (4) 13 are considered good at controlling their heart rate and (5) 8 are considered exceptional at controlling their heart rate.

Following a similar pattern than previously described we develop an immersive virtual reality game that will take advantage of the participants voluntary heart rate control as a game mechanic. The game consists in a series of 8 heart rate control exercises, one for each level in the game. The participants unlock the end of each level by reaching certain heart rate values during a defined time. From the results of the first study presented in this paper we elected the heart rate thresholds to be: (1) reaching the baseline value and lower, when reducing one's own heart rate and (2) reaching the baseline value plus 20 BPM and higher, when increasing one's own heart rate.

The game is partly inspired by Portal ${ }^{1}$. Placed in a futuristic experimental laboratory, the game consists of a succession of rooms that each possesses its unique mechanics and interaction (Figure 2). The game starts in a furnished room with a floating drone in a corner. For our mandatory biofeedback game mechanic to be adapted to each participant, we start every experiment with a calibration phase, the same one presented in the first study. The calibration takes place in the starting room, in the VR environment, during that time the participants can explore the room but are invited not to be too active. Once the calibration is over, the drone explains the context and gives the instructions to the participants.

To give feedback to the participants on their heart rate, each room has a specific interaction that is linked to it. The different

\footnotetext{
${ }^{1}$ Valve Corporation - 2007
} 
interactions are described in the next section. To induce a high connection from the participant with the virtual environment we wanted to tie the effect of heart rate to a "logical" consequence in the virtual environment [1] (e.g. the higher the heart rate the bigger the fire, the lower the heart rate the lower the volume of gas...).

From the 57 participants of the previous experiment we select 30 persons to participate in the second part of the experiment. They are classified as two competency groups for the rest of the study The "Low Control Group" (LCG) comprised of 8 participants that didn't succeed the heart rate control and 8 that are considered regular at controlling their heart rate $(\mathrm{N}=16)$. The other group, the "High Control Group" (HCG) is composed of 8 participants that are good at controlling their heart rate and 6 that are exceptional at controlling their heart rate $(\mathrm{N}=14)$. Participants experienced both difficulties of the game, in the same order. Firstly, the normal difficulty (ND) then the enhanced difficulty (ED). To study the effects of this new mechanic on user engagement we built a questionnaire evaluating different factors of engagement.
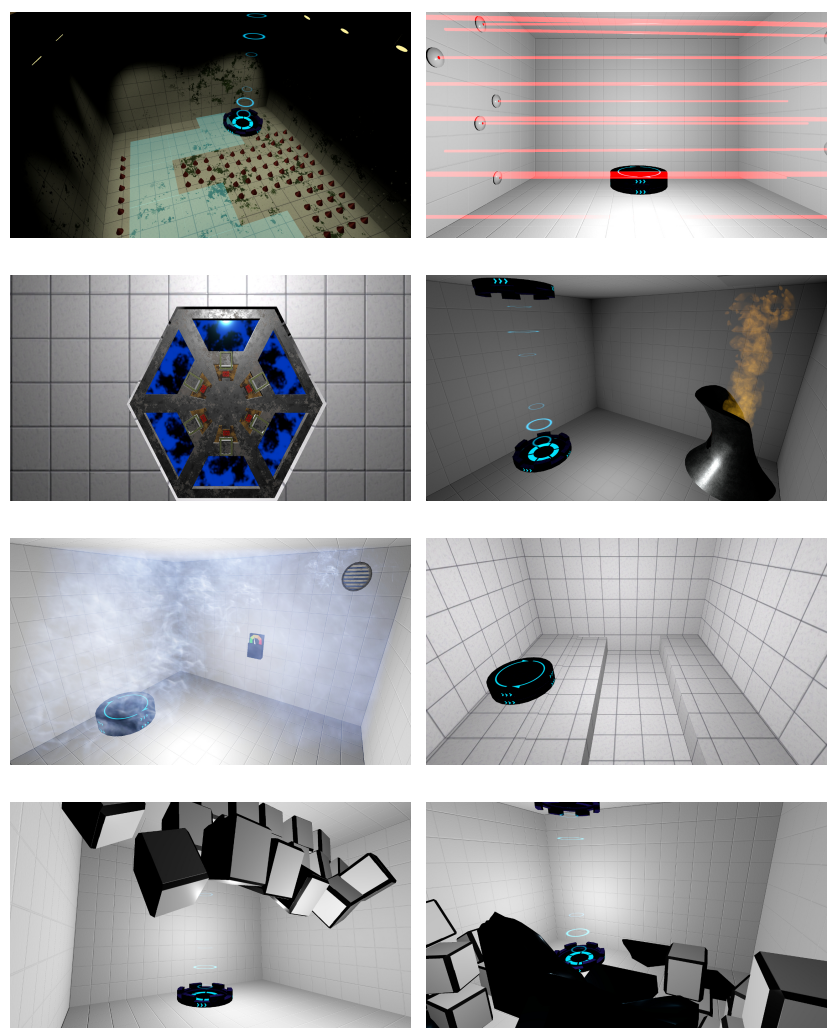

Figure 1: Illustrations of the different rooms of the VR game.

\section{Results AND discussion}

The results of our mixed between-within analysis of variance showed that there was significant interaction between the difficulty and the level of the participants for the question "How fun was the gaming experience?" (Wilks' Lambda=.759, $\mathrm{F}(1,28)=8.894, p=.006$, partial eta squared=.241). We first analyzed the results depending on the competency group for this question. When looking in detail we found no significant differences between the difficulty levels for the LCG. On the other hand, the HCG reported significantly different scores depending on the difficulty $(p=.014, \mathrm{Z}=-2.449)$, the score being higher for the second round $(\mathrm{M}(\mathrm{ED})=3.86, \mathrm{SD}=.864)$ than for the first round $(\mathrm{M}(\mathrm{ND})=3.43, \mathrm{SD}=.756)$.
We observed some significant influence of the round over the scores for questions "How successful did you feel when you overcame the obstacles in the game?" (Wilks' Lambda $=.862, \mathrm{~F}(1$, $28)=4.491, p=.043$, partial eta squared $=.138 ; \mathrm{M}(\mathrm{ND}-\mathrm{all})=3.63$, $\mathrm{SD}=1.19 ; \mathrm{M}(\mathrm{ED}-\mathrm{all})=4.10, \mathrm{SD}=.92)$ and "How much did you find that your skills were gradually improving through the course of overcoming the challenges in the game?" (Wilks' Lambda $=.830$, $\mathrm{F}(1,28)=5.723, p=.024$, partial eta squared $=.170 ; \mathrm{M}(\mathrm{ND}$-all $)=3.30$, $\mathrm{SD}=1.02 ; \mathrm{M}(\mathrm{ED}$-all $)=3.60, \mathrm{SD}=1.13)$. We note that the participants felt more rewarded during the second round. Looking into more details, using a Wilcoxon signed ranks test, these results are especially observable for the participants in the HCG, which reported significantly higher personal gratification for the questions "How much did you feel that the game constantly motivated you to proceed further to the next level?" $(p=.025, \mathrm{Z}=-2.236 ; \mathrm{M}(\mathrm{ND}-\mathrm{HCG})=3.36$, $\mathrm{SD}=.842 ; \mathrm{M}(\mathrm{ED}-\mathrm{HCG})=3.71, \mathrm{SD}=.726)$ and "How much did you find that your skills were gradually improving through the course of overcoming the challenges in the game?" $(p=.034, \mathrm{Z}=-2.121$; $\mathrm{M}(\mathrm{ND}-\mathrm{HCG})=3.29, \mathrm{SD}=.91 ; \mathrm{M}(\mathrm{ED}-\mathrm{HCG})=3.71, \mathrm{SD}=1.14)$. We can report that the participants in the HCG felt more rewarded during the ED condition as it "felt more challenging, the difficulty was more satisfying".

These results allow us to highlight that the level of engagement is indeed linked to the levels of competency and difficulty. Indeed, the participants that were rated as highly capable of influencing their heart rate reported overall better results in the different factors of engagement. Interestingly we can clearly observe that the participants in the HCG are more engaged when the difficulty of the game is higher compared to the participants in the LCG who prefer the normal difficulty.

This work presented a two-part experiment designed to study the effect of a mandatory heart rate control mechanic on user engagement in a VR experience. We were able to directly translate the results of the first part of our study into a VR experience. The usage of direct voluntary heart rate biofeedback had a significant impact on the level of engagement. The participants having better control over their heart rate reporting higher results, despite voluntary heart rate control being the only interaction mechanic.

\section{REFERENCES}

[1] C. Groenegress, B. Spanlang, and M. Slater. The physiological mirror-a system for unconscious control ofavirtual environment through physiological activity. The Visual Computer, 26(6):649-657, Jun 2010. doi: 10 .1007/s00371-010-0471-9

[2] S. Houzangbe, O. Christmann, G. Gorisse, and S. Richir. Fear as a biofeedback game mechanic in virtual reality: Effects on engagement and perceived usability. In Proceedings of the 13th International Conference on the Foundations of Digital Games, FDG '18, pp. 12:1-12:6. ACM, New York, NY, USA, 2018. doi: 10.1145/3235765.3235787

[3] K. T. Larkin, S. B. Manuck, and A. L. Kasprowicz. The effect of feedback-assisted reduction in heart rate reactivity on videogame performance. Biofeedback and Self-regulation, 15(4):285-303, Dec 1990. doi: 10.1007/BF01000024

[4] S. B. Manuck, R. W. Levenson, J. J. Hinrichsen, and S. L. Gryll. Role of feedback in voluntary control of heart rate. Perceptual and Motor Skills, 40(3):747-752, 1975. PMID: 1178359. doi: 10.2466/pms. 1975.40.3. 747

[5] M. Sra, X. Xu, and P. Maes. Breathvr: Leveraging breathing as a directly controlled interface for virtual reality games. In Proceedings of the 2018 CHI Conference on Human Factors in Computing Systems, CHI '18, pp. 340:1-340:12. ACM, New York, NY, USA, 2018. doi: 10.1145/3173574 .3173914

[6] L. A. Sroufe. Effects of depth and rate of breathing on heart rate and heart rate variability. Psychophysiology, 8(5):648-655, 1971. doi: 10. 1111/j.1469-8986.1971.tb00500.x 